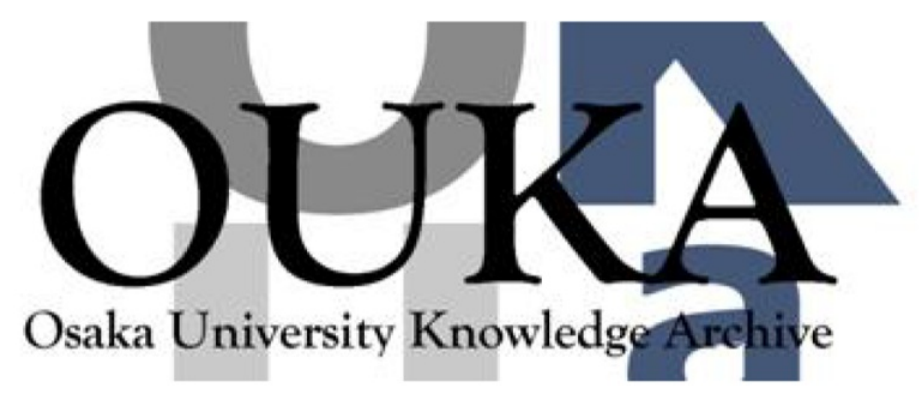

\begin{tabular}{|c|l|}
\hline Title & $\begin{array}{l}\text { Detection of nonmagnetic metal thin film using } \\
\text { magnetic force microscopy }\end{array}$ \\
\hline Author(s) & $\begin{array}{l}\text { Wakaya, Fujio; Oosawa, Kenta; Kajiwara, } \\
\text { Masahiro et al. }\end{array}$ \\
\hline Citation & Applied Physics Letters. 113(26) p. 261601 \\
\hline Issue Date & 2018-12-24 \\
\hline oaire:version AM \\
\hline URL & https://hdl. handle. net/11094/71061 \\
\hline rights & \\
\hline Note & \\
\hline
\end{tabular}

Osaka University Knowledge Archive : OUKA

https://ir. Library. osaka-u. ac. jp/

Osaka University 


\section{Detection of nonmagnetic metal thin film using magnetic force microscopy}

Cite as: Appl. Phys. Lett. 113, 261601 (2018); https://doi.org/10.1063/1.5079763

Submitted: 02 November 2018 . Accepted: 04 December 2018 . Published Online: 26 December 2018

Fujio Wakaya (Denta Oosawa, Masahiro Kajiwara, Satoshi Abo, and Mikio Takai

\section{COLLECTIONS}

EP This paper was selected as an Editor's Pick
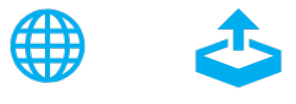

\section{ARTICLES YOU MAY BE INTERESTED IN}

Squeezing-enhanced rotating-angle measurement beyond the quantum limit Applied Physics Letters 113, 261103 (2018); https://doi.org/10.1063/1.5066028

Self-propelling Leidenfrost droplets on a variable topography surface

Applied Physics Letters 113, 243704 (2018); https://doi.org/10.1063/1.5056249

Propensity for spontaneous relaxor-ferroelectric transition in quenched $\left(\mathrm{Na}_{1} / 2 \mathrm{Bi}_{1} / 2\right) \mathrm{TiO}_{3}-$

$\mathrm{BaTiO}_{3}$ compositions

Applied Physics Letters 113, 252902 (2018); https://doi.org/10.1063/1.5053989
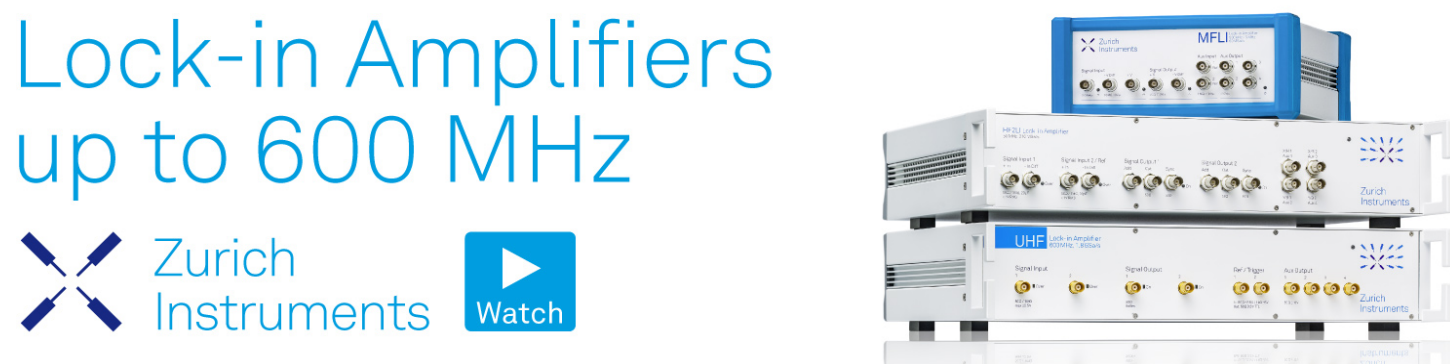


\title{
Detection of nonmagnetic metal thin film using magnetic force microscopy
}

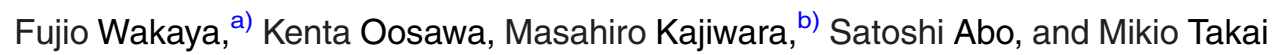 \\ Center for Science and Technology Under Extreme Conditions, Graduate School of Engineering Science, \\ Osaka University, 1-3 Machikaneyama, Toyonaka, Osaka 560-8531, Japan
}

(Received 2 November 2018; accepted 4 December 2018; published online 26 December 2018)

\begin{abstract}
Magnetic force microscopy (MFM) allows detection of stray magnetic fields around magnetic materials and the two-dimensional visualization of these fields. This paper presents a theoretical analysis of the oscillations of an MFM tip above a thin film of nonmagnetic metal. The results show good agreement with experimental data obtained by varying the tip height. The phenomenon analyzed here can be applied as a "metal detector" at the nanometer scale and for contactless measurements of sheet resistivity. The detection sensitivity is obtained as a function of oscillation frequency, thus allowing the determination of the best frequency for the phase-shift measurement. The shift in resonance frequency due to the presence of a nonmagnetic metal is also discussed. Published by AIP Publishing. https://doi.org/10.1063/1.5079763
\end{abstract}

Magnetic force microscopy (MFM) is often used for detecting stray magnetic fields near the surfaces of magnetic materials, ${ }^{1-3}$ with a magnetized tip oscillating above the material. However, in their investigation of the currentinduced magnetic field from nonmagnetic metal lines, Tanaka et al. $^{4}$ reported that MFM can detect small signals from nonmagnetic materials (see Fig. 4 of Ref. 4). They attributed the observed small signals in the absence of current to an effect of surface topography although they did not specify this effect exactly. Similar small signals have been reported by Stiller et al., ${ }^{5}$ who investigated the currentinduced magnetic field using a ring-shaped nonmagnetic metal line and observed a ring in the MFM image without any current [see Fig. 3(c) of Ref. 5]. However, neither Tanaka et al. ${ }^{4}$ nor Stiller et al. ${ }^{5}$ discussed the origin of the observed small signals in the absence of magnetic materials and of currents. In the present work, such MFM signals from a thin film of nonmagnetic metal are shown to originate from eddy currents induced in the film by the oscillating MFM tip. This effect enables us to detect the presence of a nonmagnetic metal even if it is buried in an insulator, and therefore, it could act as a "metal detector" in the nanometer region and be used for defect inspection in metal-line layers in integrated circuits. Moreover, this effect provides a contactless sheet resistivity measurement method for metallic materials, including doped semiconductors, where the dopant concentration could be measured without the need for the measurement device to come into contact with the probes.

To simplify the real MFM system, the cantilever of the device is modeled as a spring with spring constant $k$, quality factor $Q_{0}$, and mass $m$. Although the real magnetic dipole moment distributed on the MFM tip surface can be modeled as a monopole and a dipole moment located at the tip, ${ }^{6-8}$ we assume in this work a single magnetic dipole moment $p$ in the $z$ direction located at the tip end, as is often adopted as a simple model of an MFM tip. ${ }^{7}$ Figure 1 shows a schematic of the system. The equation of motion of the point mass $m$ is

\footnotetext{
${ }^{a)}$ Electronic mail: wakaya@stec.es.osaka-u.ac.jp

b)Present address: Daihatsu Motor Co., Ltd., Ikeda, Japan.
}

$$
m \ddot{z}_{\mathrm{m}}=-k\left[\left(z_{\mathrm{m}}-z_{\mathrm{m} 0}\right)-\left(u-u_{0}\right)\right]-\gamma \dot{z}_{\mathrm{m}}+F_{z},
$$

where $u$ is the $z$ position of the fixed end of the spring, $\gamma$ is the dissipation constant of the system, and $F_{z}$ is the force in the $z$ direction. The $z$ positions $z_{\mathrm{m} 0}$ and $u_{0}$ are the equilibrium positions of the point mass and the fixed end, respectively.

The force $F_{z}$ originates from the motion of the magnetic moment $p$ and is obtained as follows within the quasi-static approximation. The magnetic flux density at a circle of radius $r$ in the $x-y$ plane is

$$
B_{z}=\frac{p}{4 \pi} \frac{2 z_{\mathrm{m}}^{2}-r^{2}}{\left(z_{\mathrm{m}}^{2}+r^{2}\right)^{5 / 2}} .
$$

(SI units will be used throughout, but it should be noted that the unit of magnetic moment adopted here is $\mathrm{Wb} \mathrm{m}$, rather than $\mathrm{A} \mathrm{m}^{2}$.) The magnetic flux in a circle of radius $r$ is therefore

$$
\Phi=\int_{0}^{r} B_{z} 2 \pi r^{\prime} d r^{\prime}=\frac{p}{2} \frac{r^{2}}{\left(z_{\mathrm{m}}^{2}+r^{2}\right)^{3 / 2}} .
$$

If the magnetic moment $p$ moves with velocity $\dot{z}_{\mathrm{m}}$ in the $z$ direction, then the induced electromotive force, $-d \Phi / d t$, causes an eddy current $I$ in the metal thin film (which is located in the $x-y$ plane) such that

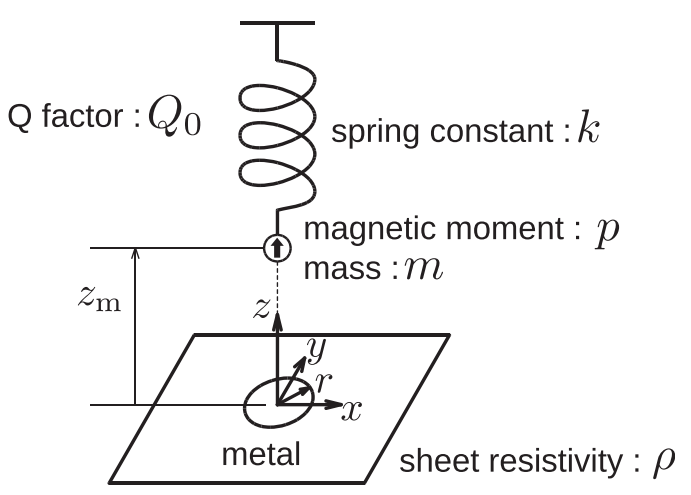

FIG. 1. Schematic of the system under consideration. 


$$
\frac{d I}{d r}=\frac{3}{4 \pi} \frac{p}{\rho} \frac{z_{\mathrm{m}} r}{\left(z_{\mathrm{m}}^{2}+r^{2}\right)^{5 / 2}} \dot{z}_{\mathrm{m}}
$$

where $\rho$ is the sheet resistivity of the metal thin film. Interaction between eddy currents at different radii is ignored in the present work. The eddy current at radius $r$ generates a magnetic field $d H_{z}$ in the $z$ direction at $(0,0, z)$ given by

$$
\frac{d H_{z}}{d r}=\frac{3}{8 \pi} \frac{p}{\rho} \frac{z_{\mathrm{m}} \dot{z}_{\mathrm{m}} r^{3}}{\left(z^{2}+r^{2}\right)^{3 / 2}\left(z_{\mathrm{m}}^{2}+r^{2}\right)^{5 / 2}} .
$$

The total magnetic field in the $z$ direction at $(0,0, z)$ is then

$$
H_{z}=\int_{0}^{\infty} \frac{d H_{z}}{d r} d r=\frac{1}{4 \pi} \frac{p}{\rho} \frac{\dot{z}_{\mathrm{m}}}{\left(z_{\mathrm{m}}+z\right)^{3}} .
$$

The force $F_{z}$ in Eq. (1) is therefore

$$
F_{z}=\left.p \frac{d H_{z}}{d z}\right|_{z=z_{\mathrm{m}}}=-\frac{3}{64 \pi} \frac{p^{2}}{\rho} \frac{\dot{z}_{\mathrm{m}}}{z_{\mathrm{m}}^{4}} .
$$

This equation shows that the direction of the force from the eddy current is opposite to that of the velocity $\dot{z}_{\mathrm{m}}$, as expected, which leads to additional dissipation and therefore a reduction in the quality factor, as discussed later.

Using Eqs. (1) and (7), the equation of motion for the oscillating MFM tip above the metal thin film is

$$
m \ddot{z}_{\mathrm{m}}=-k\left[\left(z_{\mathrm{m}}-z_{\mathrm{m} 0}\right)-\left(u-u_{0}\right)\right]-\gamma \dot{z}_{\mathrm{m}}-\frac{3}{64 \pi} \frac{p^{2}}{\rho} \frac{\dot{z}_{\mathrm{m}}}{z_{\mathrm{m}}^{4}} .
$$

The excitation of the system is assumed to be

$$
u=u_{0}+a \cos \omega t
$$

where the positive constant $a$ is the amplitude of the exciting oscillation and $\omega$ and $t$ are its angular frequency and time, respectively. In the remainder of this paper, because of the presence of a nonlinear term in Eq. (8), we adopt the smallamplitude approximation and ignore the higher harmonics. With these approximations, we can find a solution of Eq. (8) in the form

$$
z_{\mathrm{m}}=z_{\mathrm{m} 0}+A \cos (\omega t+\phi)
$$

where the positive constant $A$ is the amplitude of oscillation of the point mass $m$ and $\phi$ is the phase shift relative to the exciting oscillation. The amplitude $A$ and phase shift $\phi$ can be expressed as

$$
\begin{gathered}
A^{2}=\frac{a^{2}}{\left[1-\left(\omega / \omega_{0}\right)^{2}\right]^{2}+\left(1 / Q_{I}^{2}\right)\left(\omega / \omega_{0}\right)^{2}}, \\
\phi=-\operatorname{atan}\left[\frac{1}{Q_{I}} \frac{\omega / \omega_{0}}{1-\left(\omega / \omega_{0}\right)^{2}}\right]-\pi \theta\left(\frac{\omega}{\omega_{0}}-1\right),
\end{gathered}
$$

where $\omega_{0} \equiv \sqrt{k / m}$ and $\theta(x)$ is the step function. The modified quality factor $Q_{I}$ in Eqs. (11) and (12) is defined by

$$
\frac{m \omega_{0}}{Q_{I}} \equiv \gamma+\frac{3}{64 \pi} \frac{p^{2}}{\rho z_{\mathrm{m} 0}^{4}},
$$

which, owing to the additional dissipation, is smaller than the original quality factor $Q_{0}$ defined by

$$
\frac{m \omega_{0}}{Q_{0}} \equiv \gamma
$$

Using Eq. (12), the phase shift at the resonance frequency $\omega_{\mathrm{r}}=\omega_{0}\left[1-1 /\left(2 Q_{0}^{2}\right)\right]^{1 / 2}$ can be calculated as

$$
\phi_{\omega_{\mathrm{r}}}=-\operatorname{atan}\left[2 Q_{0}\left(1-\frac{1}{2 Q_{0}^{2}}\right)^{1 / 2}\left(1+Q_{0} \frac{z_{\mathrm{c}}^{4}}{z_{\mathrm{m} 0}^{4}}\right)\right],
$$

where the characteristic tip height $z_{\mathrm{c}}$ is defined by

$$
z_{\mathrm{c}}^{4} \equiv \frac{3}{64 \pi} \frac{\omega_{0}}{k} \frac{p^{2}}{\rho} \text {. }
$$

The phase shift above an insulator (or when the tip is far from the metal surface) is

$$
\phi_{\omega_{\mathrm{r}}}(\infty)=-\operatorname{atan}\left[2 Q_{0}\left(1-\frac{1}{2 Q_{0}^{2}}\right)^{1 / 2}\right]
$$

The phase difference between above the metal and above an insulator is therefore

$$
\Delta \phi_{\omega_{\mathrm{r}}} \equiv \phi_{\omega_{\mathrm{r}}}-\phi_{\omega_{\mathrm{r}}}(\infty) \simeq-\frac{1}{2} \frac{z_{\mathrm{c}}^{4}}{z_{\mathrm{m} 0}^{4}},
$$

where the approximation $Q_{0} \gg 1$ is used. Equation (18) represents an additional phase delay due to the eddy current in the metal.

Figure 2 shows the experimental results obtained using a 25-nm-thick Au thin film with the 1.5- $\mu$ m gap deposited on a Si substrate with 800 -nm-thick $\mathrm{SiO}_{2}$. The MFM data were recorded along a 5- $\mu \mathrm{m}$-long line across the $1.5-\mu \mathrm{m}$ gap. The additional phase delay due to the presence of the nonmagnetic metal thin film can clearly be seen. The observed phase difference is plotted as a function of $z_{\mathrm{m} 0}$ in Fig. 3. The experimentally observed phase differences are quite well fitted using Eq. (18) with $z_{\mathrm{c}}=17.4 \mathrm{~nm}$, which means that the theory presented above provides a good description of the system of MFM measurement of a nonmagnetic metal thin film. We can thus determine the characteristic tip height $z_{\mathrm{c}}$ defined in Eq. (16) by fitting the experimental data with Eq. (18). Thus, we can determine the equivalent magnetic moment of the MFM tip, $p$, if we already know the sheet resistivity of the metal, $\rho$, or we can determine $\rho$ if we have already calibrated $p$. The phase difference between above the metal and above an insulator was not observed when a tip for normal atomic force microscopy without magnetic moment was used with similar frequency, dimensions, and probe height, which means that the observed phase difference originated from the magnetic moment on the tip.

The phase difference between above the metal and above an insulator depends on the oscillation frequency $\omega$ although this is fixed at $\omega_{\mathrm{r}}$ in Eqs. (15), (17), and (18). The $\omega$ dependence of the phase shift can be written as

$$
\Delta \phi(\omega) \equiv \phi\left(\omega, z_{\mathrm{m} 0}\right)-\phi(\omega, \infty)
$$

with Eq. (12). If we define 
(a)

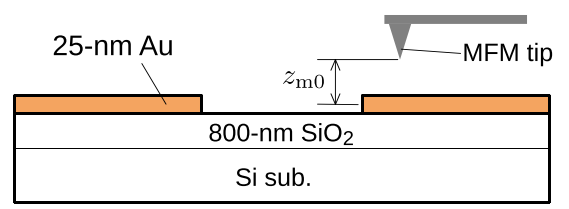

(b)

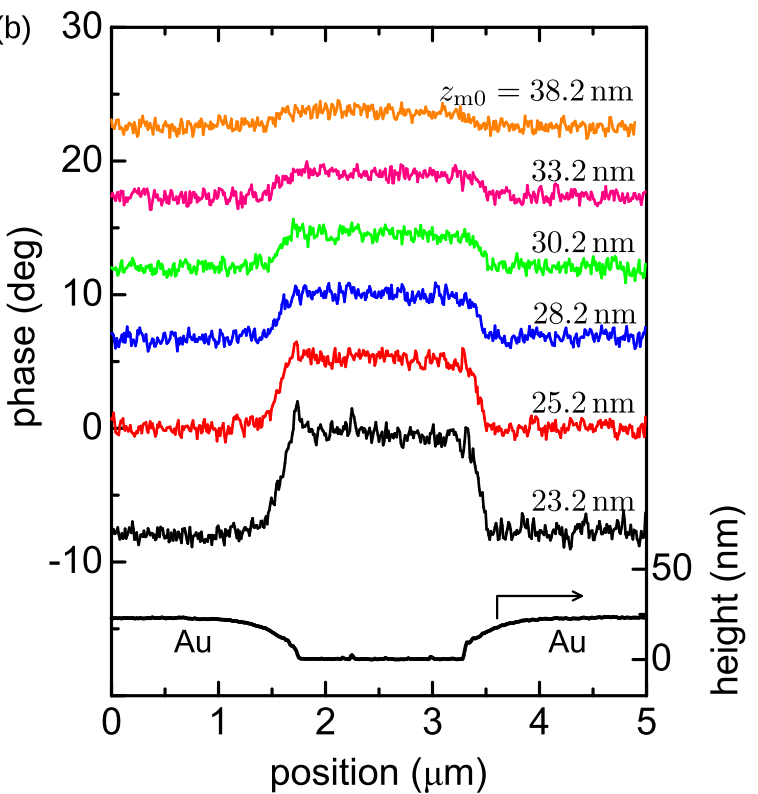

FIG. 2. (a) Cross-sectional drawing of the experimental setup. A 25-nmthick Au film with the $1.5-\mu$ m gap on 800 -nm-thick $\mathrm{SiO}_{2}$ was prepared for the experiment. (b) Experimental results for the phase and topographic height. $z_{\mathrm{m} 0}$ is measured from the center of the Au film. The lowest curve shows the topographic height, while all the other curves show the phase. The phase data are shifted vertically for ease of recognition.

$$
x \equiv \frac{z_{\mathrm{c}}^{4}}{z_{\mathrm{m} 0}^{4}},
$$

then $x$ becomes small when the tip height $z_{\mathrm{m} 0}$ becomes large or when the resistivity $\rho$ becomes large. This means that

$$
\left.S(\omega) \equiv \frac{d \Delta \phi(\omega)}{d x}\right|_{x=0}=-\frac{\frac{\omega / \omega_{0}}{1-\left(\omega / \omega_{0}\right)^{2}}}{1+\frac{1}{Q_{0}^{2}}\left[\frac{\omega / \omega_{0}}{1-\left(\omega / \omega_{0}\right)^{2}}\right]^{2}}
$$

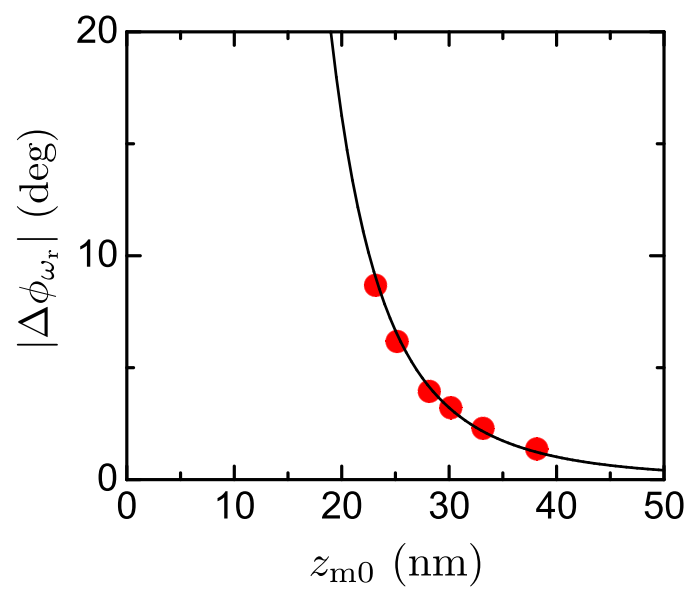

FIG. 3. Observed phase difference as a function of $z_{\mathrm{m} 0}$. The solid line shows Eq. (18) with $z_{\mathrm{c}}=17.4 \mathrm{~nm}$.

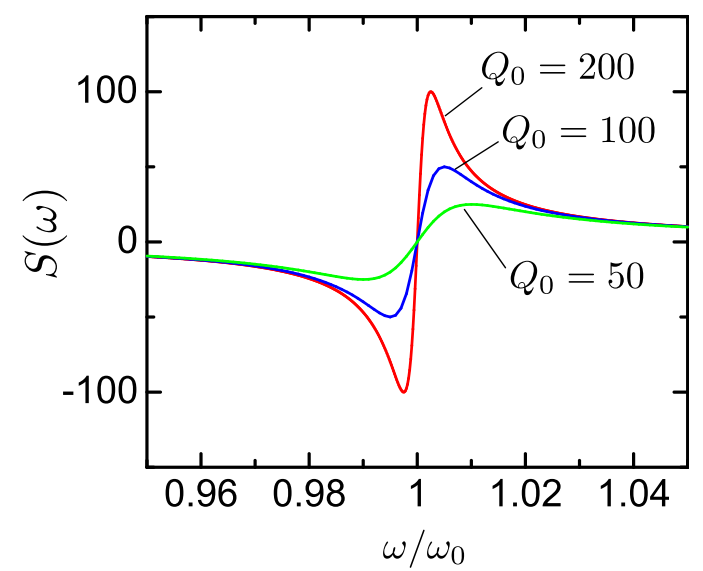

FIG. 4. Sensitivity of phase-difference detection for a large tip height and/or a high resistivity as a function of frequency.

provides the sensitivity of the phase difference as a function of frequency $\omega$ in the case of the large tip height and/or a high-resistivity metal thin film. The sensitivity $S(\omega)$ is shown in Fig. 4 for $Q_{0}=200,100$, and 50. As can be seen, the maximum sensitivity is achieved at

$$
\omega_{\max }^{ \pm} \simeq \omega_{0}\left(1 \pm \frac{1}{2 Q_{0}}\right)
$$

where the terms of higher order in $1 / Q_{0}$ are ignored, while the sensitivity becomes zero at $\omega=\omega_{0}$. At these optimum frequencies, $\omega=\omega_{\max }^{ \pm}$, the amplitude of oscillation becomes $1 / \sqrt{2}$ from the maximum, which provides a method for finding the optimum frequencies experimentally. The maximum phase difference is calculated as

$$
\Delta \phi\left(\omega_{\max }^{ \pm}\right) \simeq \pm \frac{1}{2} Q_{0} \frac{z_{\mathrm{c}}^{4}}{z_{\mathrm{m} 0}^{4}}
$$

which is a factor of $Q_{0}$ larger than $\Delta \phi_{\omega_{\mathrm{r}}}$ from Eq. (18). It is noteworthy that the phase difference changes its sign when the frequency changes across $\omega_{0}$.

The spatial resolution achieved when detecting a metal thin film using the phase shift of the MFM tip oscillation is determined by the distribution of the eddy current induced by the oscillation. From Eq. (4), the current density $J$ at a circle of radius $r$ is

$$
J \propto \frac{r / z_{\mathrm{m}}}{\left[1+\left(r / z_{\mathrm{m}}\right)^{2}\right]^{5 / 2}},
$$

as shown in Fig. 5. As can be seen, most of the eddy current is distributed within a radius $r z_{\mathrm{m}}$, which means that the detection of metal regions smaller than the tip height is difficult; in other words, the spatial resolution is roughly limited by the tip height.

Although phase shifts at a fixed frequency of oscillation have been discussed so far, the force from the eddy current induced by tip oscillation also changes the resonance frequency through additional dissipation. The resonance frequency for 


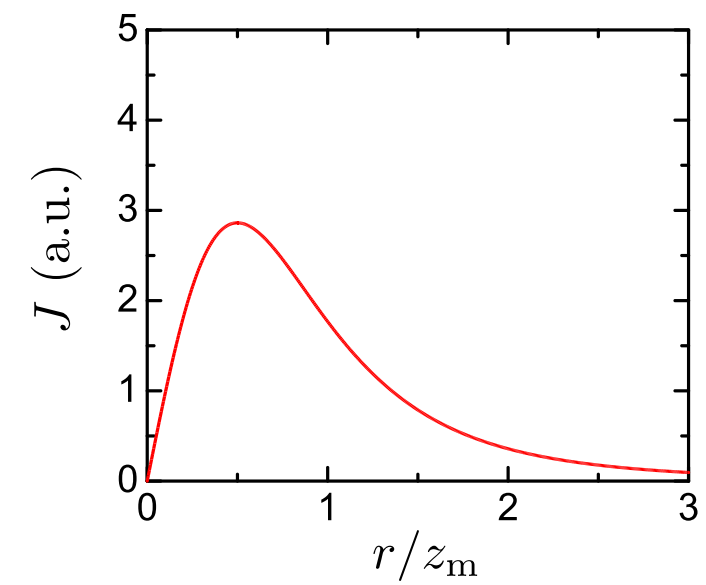

FIG. 5. Current density of eddy current as a function of radius.

$F_{z}=0$ is well known to be $\omega_{\mathrm{r}}=\omega_{0}\left[1-1 /\left(2 Q_{0}^{2}\right)\right]^{1 / 2}$. The shift in resonance frequency relative to $\omega_{\mathrm{r}}$ can be calculated as

$$
\frac{\Delta \omega}{\omega_{0}} \simeq-\frac{1}{4}\left(\frac{z_{\mathrm{c}}}{z_{\mathrm{m} 0}}\right)^{8},
$$

where the approximation

$$
\frac{1}{Q_{0}} \ll \frac{z_{\mathrm{c}}^{4}}{z_{\mathrm{m} 0}^{4}} \ll 1
$$

has been used. Such frequency shifts are often utilized in noncontact atomic force microscopy with the atomic resolution, 9,10 which means that resonance-frequency detection might have greater sensitivity than phase-shift detection for experimental detection of nonmagnetic metal.

In summary, theoretical calculations concerning an MFM tip oscillating above a nonmagnetic metal thin film have provided the phase shift due to the force from the eddy current induced by the oscillating tip. The theoretical results are in good agreement with experimental observations obtained by varying the tip height. The sensitivity of the phase shift has been shown to be a function of frequency, which allows the determination of the best frequency for the phase-shift measurement. The shift in resonance frequency due to eddy currents has also been discussed.

This work was supported by JSPS KAKENHI Grant No. $18 \mathrm{~K} 04937$.

${ }^{1}$ D. Sarid, Scanning Force Microscopy, revised ed. (Oxford University Press, Oxford, 1994).

${ }^{2}$ D. Rugar, H. J. Mamin, P. Guethner, S. E. Lambert, J. E. Stern, I. McFadyen, and T. Yogi, J. Appl. Phys. 68, 1169 (1990).

${ }^{3}$ U. Hartmann, Annu. Rev. Mater. Sci. 29, 53 (1999).

${ }^{4}$ K. Tanaka, Y. Mori, H. Yamagiwa, S. Abo, F. Wakaya, and M. Takai, Microelectron. Eng. 84, 1416 (2007).

${ }^{5}$ M. Stiller, J. Barzola-Quiquia, P. D. Esquinazi, S. Sangiao, J. M. De Teresa, J. Meijer, and B. Abel, Meas. Sci. Technol. 28, 125401 (2017).

${ }^{6}$ L. Kong and S. Y. Chou, Appl. Phys. Lett. 70, 2043 (1997).

${ }^{7}$ L. Kong and S. Y. Chou, J. Appl. Phys. 81, 5026 (1997).

${ }^{8}$ J. Lohau, S. Kirsch, A. Carl, G. Dumpich, and E. F. Wassermann, J. Appl. Phys. 86, 3410 (1999).

${ }^{9}$ H. Ueyama, M. Ohta, Y. Sugawara, and S. Morita, Jpn. J. Appl. Phys. Part II 34, L1086 (1995).

${ }^{10}$ D. Katsube and M. Abe, Appl. Phys. Lett. 113, 031601 (2018). 